\title{
Gene stacking in transgenic plants: towards compliance between definitions, terminology, and detection within the EU regulatory framework
}

\author{
Isabel TAVERNIERS ${ }^{1 * * *}$, Nina PAPAZOVA ${ }^{1 * *}$, Yves BERTHEAU $^{2}$, Marc DE LOOSE$^{1}$ and Arne HOLST-JENSEN ${ }^{3}$ \\ 1 Institute for Agricultural and Fisheries Research (ILVO), Unit Technology and Food (T\&V), Burg. Van Gansberghelaan 115 bus 2 , \\ 9820 Merelbeke, Belgium \\ 2 Institut National de la Recherche Agronomique (INRA), SPE, Centre de Versailles-Grignon, RD 10 (Route de St Cyr), 78026 Versailles \\ Cedex, France \\ ${ }^{3}$ National Veterinary Institute (NVI), Ullevaalsveien 68, P.O. Box 750 Sentrum, 0106 Oslo, Norway
}

The combination or stacking of different traits or genes in plants is rapidly gaining popularity in biotech crop production. Here we review the existing terminology regarding gene stacking in plants, and its implications in relation to genetics, biosafety, detectability and European regulations. Different methods of production of stacked gene traits, as well as the status of their cultivation and approval, are reviewed. Related to the different techniques of transformation and production, including classical breeding, and to differences in global authorization and commercialization practices, there are many types, definitions, and perceptions of stacking. These include: (1) stacking of traits and (2) stacking of events, which are the most widely accepted perceptions of stacking, and (3) stacking of genes, which from the analytical and traceability point of view may be a more appropriate perception. These differences in perceptions and definitions are discussed, as are their implications for analytical detection and regulatory compliance according to (in particular) European Union (EU) regulations. A comprehensive terminology regarding gene stacking with regulatory relevance is proposed. The haploid genome equivalent is proposed as the prevailing unit of measurement at all stages throughout the chain, in order to ensure that terminology and definitions of gene stacks are adapted to analytical detection, traceability, and compliance with EU regulations.

Keywords: transgenic / GMO / gene stacking / stacked GM plant / pyramiding / transformation / EU regulation / analytical detection

\section{INTRODUCTION}

The cultivation and breeding of biotech crops accelerated in the beginning of the second decade of their adoption. In 2007 the global area of approved biotech crops reached 114.3 million hectares, up from 102 million hectares in 2006. The principal biotech crop remains soybean (57\% of the global biotech area), followed by corn (25\% of the global biotech area), cotton (13\% of the global biotech area) and oilseed rape (5\% of the global biotech area) (James, 2007). The dominant trait during the first decade was herbicide tolerance, followed by insect protection conferred by $B t$ traits, and products combining both traits. The latter group of biotech crops showed the fastest growth $(66 \%$, in comparison with $7 \%$ growth

\footnotetext{
* Corresponding author:

isabel.taverniers@ilvo.vlaanderen.be

** These authors have contributed equally to the paper.
}

for insect resistance and 3\% for herbicide tolerance). In the existing literature, such combinations are referred to as "stacked" or "pyramided" traits. The area of cultivation of stacked traits or more precisely genetically modified organisms (GMOs) bearing stacked traits, also called "stacked GMOs", "stacked events" or simply "stacks", is expected to grow in the near future, with the introduction of new traits to meet the needs of the consumers and producers (James, 2007).

The term "gene stacking" can best be described by referring to its most common synonym, "gene pyramiding". The term gene pyramiding is used in agricultural research to describe a breeding approach to achieve pest control and higher crop yield. It is essentially a way of identifying and introducing multiple genes, which each impart resistance to an independent insect/microbial pest/weed etc., or impart resistance to a single pest through independent host pathways. This means that one might like to pyramid genes that impart resistance via different modes of 


\section{Taverniers et al.}

action to control insects, for example. Or, one might like to stack genes that impart resistance and/or tolerance to both insects and weeds, for example the combination of RoundupReady ${ }^{\circledR}(\mathrm{RR})$ and a $B t$ trait in corn or cotton. For instance, Bollgard II (registered in the U.S. in Dec. 2002) expresses two Bt proteins, Cry1A(c) and Cry2A(b)2. This is an example of genetic pyramiding for control of economically important lepidopterans on cotton. More precisely, Bollgard II in RoundupReady Flex is a triple stack, containing $\operatorname{Cry} 1 \mathrm{~A}(\mathrm{c})$ and $\mathrm{Cry} 2 \mathrm{~A}(\mathrm{~b}) 2$ genes and a RR trait. There are also transgenic corn triple stacks, for instance containing a corn root worm (CRW) protection trait (e.g., Cry3B(b)1), a corn stalk-boring insect control trait (e.g., $\operatorname{Cryl}(\mathrm{A}(b))$, and RR trait for herbicide tolerance. In this paper we will use the abbreviation "GSs" to indicate "gene stacks".

Stacked traits have been introduced into several crops via different strategies, which were reviewed previously by Halpin (2005). Halpin focused on the possibilities and drawbacks of the different strategies for multiple gene introduction into plants. We will link the different means of production to differences that exist in perceptions of stacking, types of stacks, definitions and terminology used for stacks, as well as authorization and commercialization issues. As is the case for any transgenic event, different steps precede the final authorization and commercialization of a GS. Selection of elite events is followed by appropriate cultivar production, and then by commercial seed production and propagation. Upon authorization and market introduction, processing will lead to food and feed products that contain, or are partly or completely derived from GSs and which are subject to GMO monitoring and control programs in compliance with traceability and labeling regulations. In addition to intended or commercialized gene stacks (cGSs) resulting from a desire to produce stacked GM seeds, unintended GS (uGSs) can occur when two different GMOs hybridize naturally.

Related to the different means of transformation and production of GSs, including classical cross-breeding, and to differences in global authorization and commercialization practices, different types and definitions of stacks exist. This paper aims to make the terminology regarding stacking and the resulting GSs more comprehensive, by trying to limit and group terms in relation to various implications and consequences of the definitions. Here the terminology on transformation technology and transformation events proposed by Holst-Jensen et al. (2006) is adopted as a basis. In that paper, several issues related to GMO detection and the lack of coherence with legal requirements were highlighted, but gene stacking was only discussed in part. The present paper deals with terminology and analytical detection issues specifically for different categories and types of stacked GMOs.
Within the EU, specific authorization is required for any GMO, including any GS, according to the EU GMO legislation. Key issues for approval are: (1) a case-bycase risk assessment with regard to human health and the environment (EC, 2001; 2003a); (2) availability of a validated detection method, to be provided to the Community Reference Library for interlaboratory validation; (3) availability of corresponding reference material (EC, 2004a); (4) traceability (EC, 2003a; 2003b); and (5) postmarket monitoring plans (EC, 2001). Outside the EU, other rules and practices with regard to the authorization of GSs exist.

Regulatory and analytical approaches may differ globally, with respect to how GSs are tackled. This may also have a strong impact on the possibility to define unique genetic markers for GS identification, and therefore on the detectability of GSs. The existing definitions and terms relevant to GSs are reviewed with the objective to propose a general terminology with regulatory and analytical relevance. We describe how GSs are produced, and review their cultivation and approval status. Finally, a proposal is made to facilitate coherence between terminology, analytical realities, and GMO regulations.

\section{DEFINITIONS AND TERMINOLOGY}

\section{General definition reflecting the most common perception of GSs}

The most common and general perception of gene stacking is that it refers to the combination of two or more (trans)genes of interest in the genome of the host plant, i.e. the created GMO carries two or more different new traits. Gene stacking is attractive for plant breeders wishing to introduce more than one trait into a crop plant, e.g. multiple resistances (phenotypic stacking). In transgenic plants, different genes/traits can be combined in one plant in one or in several steps. This can be achieved either by (1) direct simultaneous introduction of several different transgenes in the genome or (2) iterative processes such as retransformation or conventional crossbreeding of GM plants bearing different genetic modifications. So stacking may be achieved by more than one type of process.

The latter type of GSs, namely stacks obtained by crossing, is widely accepted by breeders, and forms the basis for the OECD definition of "unique identifier" for gene stacks (OECD, 2006). However, such a definition might be too limited, as will be discussed in details further, and it clearly implies that the term "transformation event" needs to be well defined (see also Lezaun, 2006).

Independent of modern biotechnology, "stacking" traditionally refers to the natural addition of different plant properties by genetic crossing. Modern biotechnology 
has broadened the options for stacking to include more taxonomically diverse sources, a wider selection of genes and regulatory elements, and consequently of traits.

Multiple new/enhanced crop properties are the result of the expression of different introduced "effect" genes. Halpin (2005) defined "effect" genes as ones intended to effect some useful change(s) in the final plant product, as opposed to selectable marker genes, which may only be used for the initial selection of transformed material. However, several strategies can be applied to introduce different genes into a crop, giving rise to different patterns at the genotype level of the plant. When looking at the level of the genotype, a consistent scientific definition of the terms "gene stacking" and "gene stack" (GS) and "gene stacked event" (GSE) is much more complicated. Some of the most frequently and widely used terms related to stacking are listed in Table 1.

\section{Definitions according to the transformation/ production strategy used}

When considering the different approaches for GS production, it is useful to elaborate on the terminology concerning "transformation events", proposed by Holst-Jensen et al. (2006). For the terminology regarding plant transformation products and gene stacking, we propose the acronyms listed in Table 2 , which we will also use further in this document.

Some additional remarks related to Table 2:

- It should be noted that Halpin's (Halpin, 2005) definition of "effect" genes has some limitations. In many cases, the expression of the selectable marker introduces a change in the final plant product, although the intended effect is different (e.g. corn events carrying insect resistance as the intended trait and herbicide tolerance as selectable marker).

- Holst-Jensen et al. (2006) distinguish between a StaEv and a MulEv. Stacking is thus used sensu stricto and refers to the conventional crossing of different TraEvs. In this context, stacking leads to the creation of offspring containing the inserted genetic constructs of both parental TraEvs, whereby the derived plant contains two or more physically unlinked inserted genetic constructs with independent Mendelian segregation. However, the authors remarked that from the analytical detection - and thus molecular or genotypic - point of view, there is no difference between StaEvs obtained by crossing, and MulEvs obtained by transformation with two or more transformation vectors.

- From the viewpoint of conventional breeding, i.e. when considering stacks that are produced by natural plant crosses, a "GM stacked event" is distinguished from a "GM hybrid" (De Schrijver et al., 2007; EFSA, 2006). In a GM hybrid, the transgenic trait originates from the GM inbred parental line that was crossed with one or more non-transgenic elite inbred lines. In a GM StaEv, two or more transgenic traits are brought together by crossing GM inbred lines, each being different initial events. For the latter, De Schrijver et al. (2007) define "oneway GM stacked events" as stacked events where two transgenic traits are combined, while "three-way GM stacked events" contain three transgenic traits. The current tendency, in particular in the USA, is an increasing proportion of commercial four-way stacked events, while current developments go up to nine-way stacked events.

For the production of GSs, Halpin (2005) distinguished multiple introductions of single transgene constructs from single introduction of multiple transgenes. Iterative strategies refer to the sequential/multiple introduction of single-transgene constructs into one plant. This can be achieved by (a) crossing plants containing single transgenes, or (b) retransformation of a single-transgene plant with additional transgenes. Examples of GSs produced by crossing are current corn and cotton events conferring herbicide tolerance and insect resistance, with a current introduction of up to four transgenes in a final breeders' seed. For cross-breeding-derived GSs, it may, as mentioned earlier, be relevant to discriminate between cGSs and uGSs. Examples of GSs produced by retransformation are cotton event 15985 with enhanced insect resistance, and potato events that include genes coding for both insect and virus resistance.

Cotransformation methods are used for the simultaneous introduction of multiple constructs into one plant. One method is by transformation with a plasmid construct carrying several genes of interest, a growing practice in biotech companies, i.e. assembling multigenic cassettes and introducing these multiple genes of interests on different transgene constructs. It can also be achieved by cotransformation with different plasmids or multiple DNA fragments carrying different transgenes, introduced into the plant cell either via Agrobacterium-mediated transformation or biolistic methods. By this strategy the transgenes tend to insert in a single locus, although there are some cases with multiple loci insertions (e.g. corn event Mon832).

Examples of GSs that were produced by cotransformation are corn stacks containing both herbicide tolerance and insect resistance traits or multiple insect resistances (see Tabs. 3 and 4) and some non-commercialized rice stacks conferring multiple insect resistance (Cummins, 2004).

The different ways by which GSs can be produced or occur are summarized in Figures 1,2 and 3. Figure 4 


\section{Taverniers et al.}

Table 1. Summary of terminology used for gene stacks, relative to the process of production and to the resulting event (modified from Halpin, 2005).

\begin{tabular}{|c|c|c|}
\hline Resulting event & Process of production & Definition \\
\hline $\begin{array}{l}\text { Gene stack (GS) } \\
\text { Stack } \\
\text { Stacked trait }\end{array}$ & \multirow[t]{2}{*}{ Stacking } & $\begin{array}{l}\text { GM plant combining two or more } \\
\text { genes/traits in its genome and } \\
\text { produced by conventional } \\
\text { crossing. This definition clearly } \\
\text { refers to the plant phenotype. }\end{array}$ \\
\hline $\begin{array}{l}\text { Stacked event } \\
\text { Gene-stacked event } \\
(\text { GSEv) }\end{array}$ & & $\begin{array}{l}\text { GM plant combining two or more } \\
\text { genes/traits and produced by } \\
\text { conventional crossing, } \\
\text { retransformation, or } \\
\text { cotransformation. The term event } \\
\text { refers to the introduced } \\
\text { genes/traits and its unique } \\
\text { integration pattern in the plant } \\
\text { genome. This definition refers to } \\
\text { the plant genotype. }\end{array}$ \\
\hline $\begin{array}{l}\text { Pyramided traits } \\
\text { Pyramided events } \\
\text { (equivalent to stacking) }\end{array}$ & Pyramiding & Similar to stacking \\
\hline $\begin{array}{l}\text { Multi-trait or combined-trait } \\
\text { event }\end{array}$ & Cotransformation & $\begin{array}{l}\text { GM plant which contains more } \\
\text { than one gene/trait } \\
\text { simultaneously introduced in the } \\
\text { plant genome via transformation } \\
\text { with two vectors. The transgenic } \\
\text { inserts tend to integrate in a } \\
\text { single locus, although exceptions } \\
\text { are not uncommon. }\end{array}$ \\
\hline $\begin{array}{l}\text { Multi-trait or combined-trait } \\
\text { event with separate inserts }\end{array}$ & $\begin{array}{l}\text { Multiple or } \\
\text { re-transformation }\end{array}$ & $\begin{array}{l}\text { GM plant produced by iterative } \\
\text { transformation with vectors } \\
\text { containing different } \\
\text { transgenes/traits. The transgenic } \\
\text { inserts are integrated in multiple } \\
\text { loci. }\end{array}$ \\
\hline $\begin{array}{l}\text { Co- or multiple } \\
\text { transformants obtained } \\
\text { from concomitant or } \\
\text { successive insert } \\
\text { introductions }\end{array}$ & Multigene introduction & $\begin{array}{l}\text { Introduction of multiple } \\
\text { genes/traits, each with its own } \\
\text { regulatory elements, via single or } \\
\text { cotransformation. The } \\
\text { transformations each result in a } \\
\text { separate integration locus. }\end{array}$ \\
\hline
\end{tabular}

summarizes the different perceptions of stacking and types of stacks. Phenotypic stacking refers to the presence of multiple traits and thus, following the terminology of Table 2, multiple ModSeqs. Seen from the broader genotypic viewpoint, however, stacking can be either the presence of multiple events, or of multiple genes (not necessarily corresponding to different ModSeqs). Given this broader definition, the term stacking refers to any process or situation where multiple genes are present in one plant genome, albeit as a result of direct transformation (first level), or of conventional crossing of first-level transformation events (second level) (Fig. 4).

\section{Definitions related to risk assessment and detection/identification of GSs}

The European Food Safety Authority (EFSA) opinion on the risk assessment of plants containing genetic modifications combined by crossing, considers "stacked events" 
Table 2. List of acronyms, terms and definitions (modified from Holst-Jensen et al., 2006) and proposed as common terminology regarding GSs, with regulatory relevance.

\begin{tabular}{|c|c|c|}
\hline Acronym & Term & Definition \\
\hline \multirow[t]{3}{*}{ ModSeq } & Modified sequence & $\begin{array}{l}\text { The result at the molecular level, i.e. insertion of } \\
\text { new/modified genetic information in the genome of the } \\
\text { modified cell/organism (limited to a single functional } \\
\text { genetic construct, also covering vector elements and } \\
\text { non-expressed genes, possible rearrangements, } \\
\text { insertions/deletions, or substitutions of a part of the } \\
\text { genome of the cell) }\end{array}$ \\
\hline & (Gene) Construct & $\begin{array}{l}\text { A combination of genetic elements serving the purpose } \\
\text { of activation and regulation of the expression of the } \\
\text { gene which is part of the construct, after integration in } \\
\text { the genome of the recipient organism (not including } \\
\text { vector elements and non-expressed genes) }\end{array}$ \\
\hline & Transformation & $\begin{array}{l}\text { The process of modification of a cell by the uptake and } \\
\text { incorporation of exogenous (foreign) DNA, and leading } \\
\text { to the establishment of one or more ModSeqs in the } \\
\text { modified cell }\end{array}$ \\
\hline TraEv & Transformation event & $\begin{array}{l}\text { The result of transformation at the plant level, defined } \\
\text { as the primary transformant, which is always } \\
\text { hemizygous for the ModSeq }\end{array}$ \\
\hline InSeq & Integrated sequence & $\begin{array}{l}\text { The inserted new/modified genetic construct(s), also } \\
\text { including non-functional/non-expressed inserts, such as } \\
\text { partial/truncated constructs }\end{array}$ \\
\hline UniEv & Unique event & $\begin{array}{l}\text { A single event, carrying a single-copy single function } \\
\text { ModSeq, or a ModSeq at one locus }\end{array}$ \\
\hline EliEv & Elite event & $\begin{array}{l}\text { TraEvs with optimal trait performance, subjected to } \\
\text { further authorization and marketing of the newly } \\
\text { produced transgenic event }\end{array}$ \\
\hline MulEv & Multiple event & $\begin{array}{l}\text { An event carrying multiple-copy and/or multiple } \\
\text { functional ModSeq, at one locus or at different loci }\end{array}$ \\
\hline StaEv & Stacked event & $\begin{array}{l}\text { Is obtained by conventional crossing between single } \\
\text { EliEvs, i.e. the result of cross-hybridization between two } \\
\text { previously independent events, where the progeny } \\
\text { carries at least one ModSeq from each of the parent } \\
\text { events }\end{array}$ \\
\hline
\end{tabular}

as F1 hybrids between two elite lines each containing a single transgenic event. In a general definition, the term "hybrid" covers the F1 generation of two genetically different plants, lines, cultivars, subspecies, species or genera, including two different transgenic lines (EFSA, 2006). The risk assessment of GSs with existing evaluation of the parental lines should focus on the following items: assessment of the integrity of the transgene loci in the GS, phenotypic stability, and assessment of the potential interactions between the combined events. Stacking by retransformation is considered by EFSA as a different scenario, since a completely new transgene locus is involved, and it is obviously recommended that these events be treated as primary transformants for risk assessment purposes (EFSA, 2004).

Following the terminology in Table 2, the term "TraEv" is used for any event that is the direct result of transformation, while "StaEv" is used for hybrids obtained by crossing two parental TraEvs. Given the different strategies by which GSs can be produced, and when applying this distinction, StaEvs can also be TraEvs. This means that from the molecular/genotypic point of 
Table 3. Overview of stacked gene events. Summary of the transgenic events that received authorization worldwide, their method of production, introduced ModSeqs, traits (effect genes) and their copy number and loci number. The summary is based on data from the AgBios database, BATs reports, the EU database.

\begin{tabular}{|c|c|c|c|c|c|}
\hline Event & Method of production/ & Traits & $\begin{array}{l}\text { Introduced } \\
\text { effect genes }\end{array}$ & $\begin{array}{l}\text { Effect genes copy } \\
\text { number }\end{array}$ & Loci number \\
\hline \multicolumn{6}{|c|}{ Corn } \\
\hline \multicolumn{6}{|c|}{ Introduction of one T-DNA insert (ModSeq) containing linked effect genes } \\
\hline \multirow[t]{2}{*}{ Bt11 } & Direct DNA transfer & LRes & CrylAb & 1 & 1 \\
\hline & & HT(Glu) & pat & & \\
\hline \multirow[t]{2}{*}{676} & Particle bombardment & MS & Dam & 1 & $>1 ?$ \\
\hline & & HT(Glu) & pat & 2 & \\
\hline \multirow[t]{2}{*}{678} & Particle bombardment & MS & Dam & 3 & $>1 ?$ \\
\hline & & HT(Glu) & pat & 2 & \\
\hline \multirow[t]{2}{*}{680} & Particle bombardment & MS & Dam & 4 & $>1 ?$ \\
\hline & & HT(Glu) & pat & 1 & \\
\hline \multirow[t]{2}{*}{ TC1507 } & Particle bombardment & LRes & CrylFa2 & $\geq 1$ & 1 \\
\hline & & HT(Glu) & pat & 1 & \\
\hline \multirow[t]{2}{*}{ Mon88017 } & Agrobacterium-mediated transformation/1 & LRes & Cry $3 \mathrm{Bbl}$ & 1 & 1 \\
\hline & & HT(Gly) & EPSPS & & \\
\hline \multirow[t]{2}{*}{ MON89034 } & Agrobacterium-mediated transformation & LRes & CrylA.105 & 1 & 1 \\
\hline & & & Cry $2 A b 2$ & & \\
\hline \multirow[t]{2}{*}{ DAS-062758 } & Agrobacterium-mediated transformation & LRes & CrylF & 1 & 1 \\
\hline & & HT(Glu) & bar & & \\
\hline \multirow[t]{3}{*}{ DAS59122 } & Agrobacterium-mediated transformation & DLRes & Cry34Ab1 & 1 & 1 \\
\hline & & & Cry35Ab1 & & \\
\hline & & HT(Glu) & pat & & \\
\hline \multirow{3}{*}{ Bt176 } & Simultaneous introduction of tw & NA inserts & erring differe & ct genes & \\
\hline & Cotransformation via particle bombardments & LRes & CrylAb & $>2$ & $>2$ (linked) \\
\hline & & HT(Glu) & bar & & \\
\hline \multirow[t]{2}{*}{ CBH351 } & Cotransformation by particle bombardment & LRes & Cry9C & 1 & 1 \\
\hline & & HT(Glu) & bar & 4 & \\
\hline \multirow[t]{2}{*}{ DBT418 } & Cotransformation via particle bombardment & LRes & $\operatorname{CrylA}(c)$ & 2 & 1 \\
\hline & & HT(Glu) & bar & 1 & \\
\hline \multirow[t]{2}{*}{ Mon801 } & Cotransformation via particle bombardment & LRes & CrylAb & 2 & 2 \\
\hline & & HT(Gly) & CP4 EPSPS & 2 and 1 partial & \\
\hline
\end{tabular}


Table 3. Continued.

\begin{tabular}{|c|c|c|c|c|c|}
\hline Event & Method of production/ & Traits & $\begin{array}{l}\text { Introduced } \\
\text { effect genes }\end{array}$ & $\begin{array}{l}\text { Effect genes copy } \\
\text { number }\end{array}$ & Loci number \\
\hline \multirow[t]{2}{*}{ Mon802 } & Cotransformation via particle bombardment & LRes & CrylAb & 1 & 1 \\
\hline & & HT(Gly) & CP4 EPSPS & 1 & \\
\hline \multirow[t]{2}{*}{ Mon809 } & Cotransformation via particle bombardment & LRes & CrylAb & 2 & 1 \\
\hline & & HT(Gly) & CP4 EPSPS & 2 & \\
\hline \multirow[t]{2}{*}{ MON832 } & Cotransformation via particle bombardment/2 & LRes & CrylAb & 0 & 1 \\
\hline & & HT(Gly) & CP4 EPSPS & 2 & \\
\hline MS3 & Cotransformation & MS & $\begin{array}{l}\text { Barnase } \\
\text { barstar }\end{array}$ & 1 & 1 \\
\hline \multirow[t]{2}{*}{ MS6 } & Cotransformation & MS & $\begin{array}{l}\text { Barnase } \\
\text { bar }\end{array}$ & 1 & 1 \\
\hline & & $\mathrm{HT}(\mathrm{Glu})$ & & & \\
\hline & $\begin{array}{l}\text { Sequential introductiol } \\
\end{array}$ & e than on & gene & & \\
\hline \multirow{2}{*}{$\begin{array}{l}\text { ACSZM003-2 } \times \\
\text { MON810 }\end{array}$} & F1 hybrid of T25 and Mon 810 & LRes & CrylAb & 1 & 2 \\
\hline & & HT(Glu) & pat & 1 & \\
\hline \multirow[t]{2}{*}{ DAS1507 $\times$ MON603 } & F1 hybrid of NK603 and TC1507 & LRes & CrylFa2, & $\geq 1$ & 2 \\
\hline & & HT(Glu) & PAT & 1 & \\
\hline MON603 $\times$ MON810 & & HT(Gly) & CP4 EPSPS & 1 & \\
\hline \multirow[t]{2}{*}{ MON863 × MON810 } & F1 hybrid of Mon863 and Mon810 & DLRes & crylAb & 1 & 2 \\
\hline & & & $\operatorname{cry} 3 B b 1$ & 1 & \\
\hline MON863 $\times$ MON810 $\times$ & F1 hybrid of stacked line Mon863 $\times$ Mon810 and & DLRes & CrylAb & 1 & 3 \\
\hline \multirow[t]{2}{*}{ MON603 } & NK603 & & Cry $3 B b 1$ & 1 & \\
\hline & & HT(Gly) & CP4 EPSPS & 1 & \\
\hline \multirow[t]{2}{*}{ GA21 $\times$ MON810 } & F1 hybrid of GA21 and Mon810 & LRes & CrylAb & 1 & 2 \\
\hline & & HT(Gly) & CP4 EPSPS & 1 & \\
\hline \multirow[t]{2}{*}{ NK603 $\times$ Mon863 } & F1 hybrid of Mon863 and NK603 & DLRes & Cry3Bb1 & 1 & 2 \\
\hline & & HT(Gly) & CP4 EPSPS & 1 & \\
\hline \multirow[t]{4}{*}{ TC1507 × AS59122 } & F1 hybrid of TC1507 and DAS59122 & TLRes & CrylFa2 & $\geq 1$ & 2 \\
\hline & & & cry $34 A b 1$ & 1 & \\
\hline & & & cry35Ab1 & 2 & \\
\hline & & HT(Glu) & pat & 1 & \\
\hline \multirow[t]{4}{*}{ DAS59122 × NK603 } & F1 hybrid of DAS59122 and NK603 & DLRes & cry34Ab1 & 1 & 2 \\
\hline & & & cry $35 A b 1$ & 1 & \\
\hline & & HT(Gly) & CP4 EPSPS & 1 & \\
\hline & & HT(Glu) & pat & 1 & \\
\hline \multirow{3}{*}{$\begin{array}{l}\text { DAS59122 } \times \text { TC1507 } \times \\
\text { NK603 }\end{array}$} & & & cry $35 A b 1$ & 1 & \\
\hline & & HT(Glu) & pat & 2 & \\
\hline & & HT(Gly) & CP4 EPSPS & 1 & \\
\hline
\end{tabular}


Table 3. Continued.

\begin{tabular}{|c|c|c|c|c|c|}
\hline Event & Method of production/ & Traits & $\begin{array}{l}\text { Introduced } \\
\text { effect genes }\end{array}$ & $\begin{array}{l}\text { Effect genes copy } \\
\text { number }\end{array}$ & Loci number \\
\hline \multirow[t]{2}{*}{ MON810 × LY038 } & \multirow[t]{2}{*}{ F1 hybrid of MON810 and LY038 } & LRes & CrylA & 1 & \multirow[t]{2}{*}{2} \\
\hline & & Lys & cordapA & 1 & \\
\hline \multirow[t]{3}{*}{ MON810 × MON88017 } & \multirow[t]{3}{*}{ F1hybrid of MON810 and MON88017 } & CRes & CrylA & 1 & \multirow[t]{3}{*}{2} \\
\hline & & LRes & Cry $3 B b 1$ & 1 & \\
\hline & & HT(Gly) & CP4 EPSPS & 1 & \\
\hline \multirow[t]{2}{*}{ Bt11 × GA21 } & \multirow[t]{2}{*}{ F1 hybrid of Bt11 and GA21 } & LRes & CrylAb & 1 & \multirow[t]{2}{*}{2} \\
\hline & & HT(Glu) & Pat & 1 & \\
\hline \multirow[t]{3}{*}{ MON89034 × NK603 } & \multirow[t]{3}{*}{ F1 hybrid of MON89034 and NK603 } & \multirow[t]{2}{*}{ LRes } & Cry11.105 & 1 & \\
\hline & & & $\operatorname{Cry} 2 \mathrm{Ab} 2$ & 1 & \\
\hline & & HT(Gly) & CP4 EPSPS & 1 & \\
\hline \multirow[t]{4}{*}{ MON89034 × MON88017 } & \multirow[t]{4}{*}{ F1 hybrid of MON89034 and MON88017 } & \multirow[t]{2}{*}{ LRes } & Cry11.105 & 1 & \multirow[t]{4}{*}{2} \\
\hline & & & Cry $2 A b 2$ & 1 & \\
\hline & & CRes & Cry $3 B b 1$ & 1 & \\
\hline & & HT(Gly) & CP4 EPSPS & 1 & \\
\hline \multicolumn{6}{|c|}{$\begin{array}{l}\text { Cotton } \\
\end{array}$} \\
\hline $19-51$ & Agrobacterium-mediated transformation & HT(sulf) & Chimeric $\mathrm{S} 4-\mathrm{HrA}$ & 2 & 1 \\
\hline $531,757,1076$ & Agrobacterium-mediated transformation & LRes & CrylAc & 2 & 2 \\
\hline DAS-24236-5 (281-24-) & \multirow[t]{2}{*}{ Agrobacterium-mediated transformation } & LRes & CrylF & 1 & \multirow[t]{2}{*}{1} \\
\hline 236 & & HT(Glu)-SM & pat & 1 & \\
\hline DAS-21023-5 (3006-210- & \multirow[t]{2}{*}{ Agrobacterium-mediated transformation } & LRes & CrylAc & 1 & \multirow[t]{2}{*}{1} \\
\hline 23) & & HT(Glu)-SM & pat & 1 & \\
\hline $23-198$ & Agrobacterium-mediated transformation & FAC & Bay TE & 3 & 1 \\
\hline $23-18-17$ & Agrobacterium-mediated transformation & FAC & Bay TE & 15 & 5 \\
\hline Falcon GS/40/90 & Agrobacterium-mediated transformation & HT(Gly & pat & 2 & 2 \\
\hline PHY14, PHY35 & \multirow[t]{2}{*}{ Agrobacterium-mediated transformation } & HT(Glu) & Bar & \multirow[t]{2}{*}{ No information available } & \multirow{2}{*}{$\begin{array}{l}\text { No information } \\
\text { available }\end{array}$} \\
\hline PHY36 & & MS & $\begin{array}{l}\text { Barnase } \\
\text { barstar }\end{array}$ & & \\
\hline HCN92 (Topas 19/2) & Agrobacterium-mediated transformation & bar & pat & 2 & 1 \\
\hline & Sequential introductio & of more than 0 & e effect gene & & \\
\hline 15985 & Retransformation via particle bombardment & DLRes & Cry $2 A b$ & 1 & 1 \\
\hline & & & CrylAc & 2 & 1 \\
\hline DAS2103-5 $\times$ & F1 hybrid of DAS2103-5 and DAS24236-5 & LRes & CrylF, & 1 & 2 \\
\hline DAS-21023-5 $\times$ & F1 hybrid of DAS2103-5 $\times$ DAS24236-5 and & LRes & CrylF & 1 & 3 \\
\hline DAS-24236-5 $\times$ & MON-01445-2 & CrylAc & 1 & & \\
\hline MON-01445-2 & & HT(Glu)-SM? & pat & 2 & \\
\hline & & HT(Gly) & CP4 EPSPS & 1 & \\
\hline
\end{tabular}


Table 3. Continued.

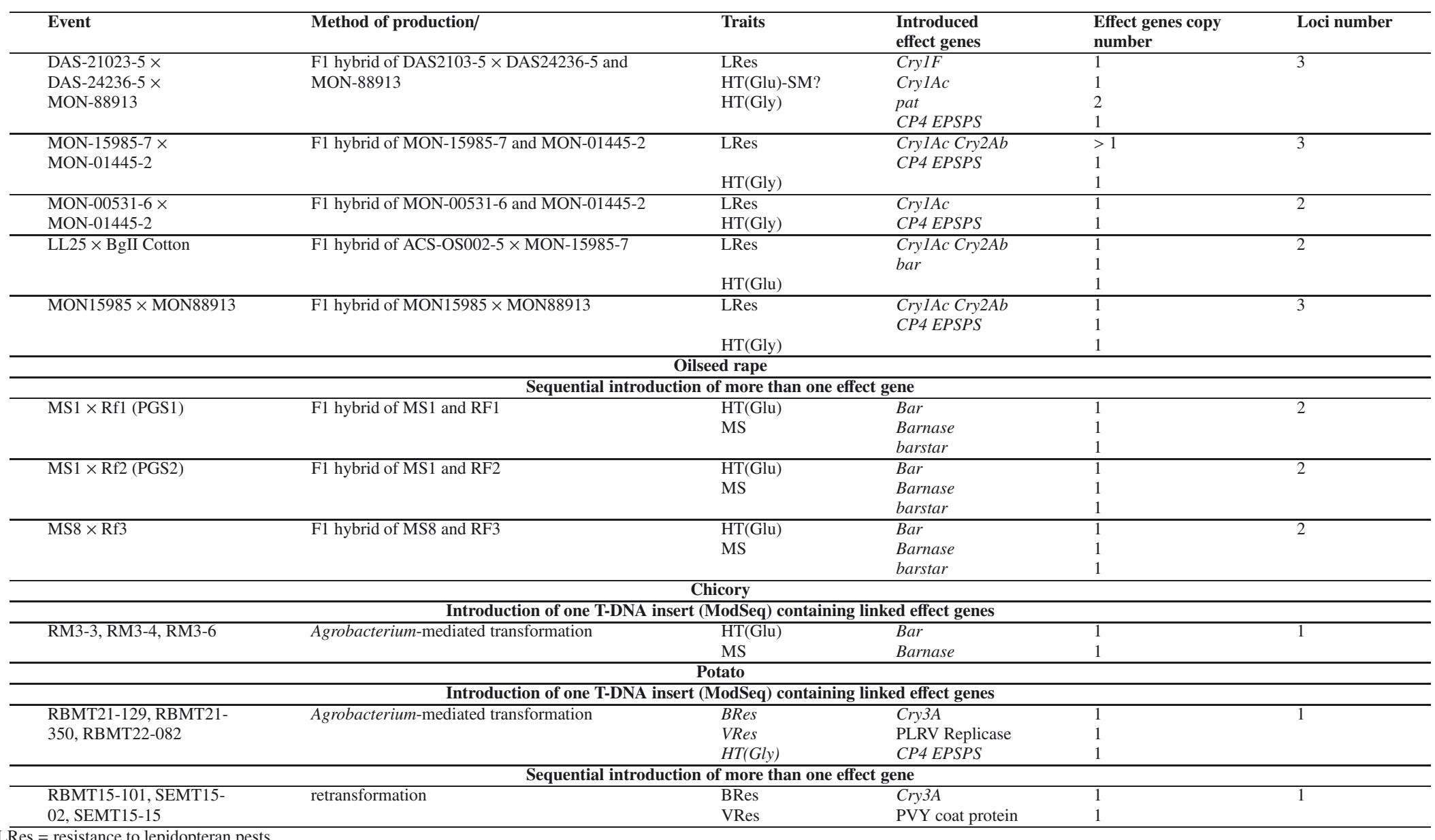

LRes = resistance to lepidopteran pests.

CRes $=$ resistance to coleopteran pests.

$\mathrm{EL} / \mathrm{CRes}=$ enhanced resistance to lepidopteran and/or coleopteran pests.

$\mathrm{HT}(\mathrm{Glu})=$ tolerance to gluphosinate ammonium herbicides.

$\mathrm{HT}(\mathrm{Gly})=$ tolerance to glyphosate herbicides

$\mathrm{HT}($ sulf $)=$ tolerance to sulfuronyl herbicides.

Lys $=$ enhanced lysine level.

MS = male sterility/fertility restorer.

$\mathrm{SM}=$ selectable maker.

$\mathrm{FAC}=$ modified fatty acid content.

BRes $=$ resistance to Colorado beetle.

VRes $=$ virus resistance. 
I. Taverniers et al.

Table 4. Authorization status of GSs produced by combining two single transgenic events (sources: AgBios database, BATs reports, EU database, U.S. Regulatory Agencies Unified Biotechnology Website).

\begin{tabular}{|c|c|c|}
\hline Crop/event & $\begin{array}{l}\text { Regulatory } \\
\text { approvals }\end{array}$ & Proposed use \\
\hline \multicolumn{3}{|c|}{ Corn } \\
\hline ACSZM003-2 $\times$ & Japan & Food/feed \\
\hline MON810 & USA* & Food/feed, environment \\
\hline \multirow[t]{5}{*}{ DAS1507 × MON603 } & Japan, & Food/feed, environment \\
\hline & Korea & Food \\
\hline & Philippines & Food/feed \\
\hline & EU & Food/feed \\
\hline & $\mathrm{USA}^{*}$ & Food/feed, environment \\
\hline \multirow[t]{6}{*}{ MON603 × MON810 } & Japan & Food/feed \\
\hline & Korea & Food \\
\hline & Mexico & Food \\
\hline & Philippines & Food/feed \\
\hline & EU & Food/feed \\
\hline & USA* & Food/feed, environment \\
\hline \multirow[t]{5}{*}{ MON863 $\times$ MON810 } & Japan & Food/feed, \\
\hline & Korea & Food \\
\hline & Philippines & Food/feed \\
\hline & EU & Feed \\
\hline & USA* & Food/feed, environment \\
\hline \multirow[t]{4}{*}{ MON863 $\times$ MON810 $\times$ MON603 } & Japan & Food/feed, \\
\hline & Philippines & Food/feed \\
\hline & $\mathrm{EU} * *$ & \\
\hline & USA* & Food/feed, environment \\
\hline \multirow[t]{6}{*}{ GA21 $\times$ MON-810 } & Japan & Food/feed \\
\hline & Korea & Food \\
\hline & Philippines & Food/feed \\
\hline & South Africa & Food/feed \\
\hline & EU & Food/feed \\
\hline & USA* & Food/feed, environment \\
\hline \multirow[t]{6}{*}{ NK603 × Mon863 } & Japan & Food/feed \\
\hline & Korea & Food \\
\hline & Mexico & Food \\
\hline & Philippines & Food/feed \\
\hline & EU & Food/feed \\
\hline & USA $*$ & Food/feed, environment \\
\hline \multirow{4}{*}{ TC1507 × AS59122 } & Japan & Food/feed \\
\hline & Korea & Food \\
\hline & $\mathrm{EU}^{* *}$ & \\
\hline & USA* & Food/feed, environment \\
\hline \multirow[t]{4}{*}{ DAS59122 × NK603 } & Japan & Food/feed \\
\hline & Korea & Food \\
\hline & Philippines & Food/feed \\
\hline & $\mathrm{USA}^{*}$ & Food/feed, environment \\
\hline \multirow[t]{3}{*}{ DAS59122 × TC1507 × NK603 } & Japan & Food/feed \\
\hline & Korea & Food \\
\hline & $\mathrm{USA}^{*}$ & Food/feed, environment \\
\hline \multirow[t]{2}{*}{ MON810 × LY038 } & Philippines & Food/feed \\
\hline & $\mathrm{USA}^{*}$ & Food/feed, environment \\
\hline \multirow[t]{3}{*}{ MON810 × MON88017 } & Japan & Food \\
\hline & Philippines & Food/feed \\
\hline & $\mathrm{USA}^{*}$ & Food/feed, environment \\
\hline
\end{tabular}


Table 4. Continued.

\begin{tabular}{|c|c|c|}
\hline Crop/event & $\begin{array}{l}\text { Regulatory } \\
\text { approvals }\end{array}$ & Proposed use \\
\hline \multirow[t]{2}{*}{ Bt11 × GA21 } & Korea & Food \\
\hline & USA* & Food/feed, environment \\
\hline \multirow[t]{2}{*}{ MON89034 × NK603 } & $\mathrm{EU} * *$ & \\
\hline & USA* & Food/feed, environment \\
\hline MON89034 × MON88017 & $\mathrm{EU} * *$ & \\
\hline \multicolumn{3}{|c|}{ Cotton } \\
\hline \multirow[t]{6}{*}{ DAS2103-5 × DAS24236-5 } & Australia & Food \\
\hline & Japan & Food/feed \\
\hline & Korea & Food \\
\hline & Mexico & Food \\
\hline & USA & Food/feed, environment \\
\hline & $\begin{array}{l}\text { EU - pending } \\
\text { authorization }\end{array}$ & \\
\hline \multirow[t]{3}{*}{ DAS-21023-5 × DAS-24236-5 × MON-01445-2 } & Japan & Food/feed \\
\hline & Mexico & Food \\
\hline & USA* & Food/feed, environment \\
\hline \multirow[t]{2}{*}{ DAS-21023-5 × DAS-24236-5 × MON-88913 } & Japan & Food/feed \\
\hline & USA $*$ & \\
\hline \multirow[t]{6}{*}{ MON-15985-7 × MON-01445-2 } & Australia & Environment \\
\hline & Japan & Food/feed \\
\hline & Korea & Food \\
\hline & Philippines & Food/feed \\
\hline & $\mathrm{EU} * *$ & Food/feed \\
\hline & $\mathrm{USA}^{*}$ & Food/feed, environment \\
\hline \multirow[t]{7}{*}{ MON-00531-6 × MON-01445-2 } & Australia & Environment \\
\hline & Japan & Food/feed \\
\hline & Korea & Food \\
\hline & Mexico & Food \\
\hline & Philippines & Food/feed \\
\hline & EU & Food/feed \\
\hline & USA* & Food/feed, environment \\
\hline \multirow[t]{2}{*}{ LL25 × BgII Cotton } & Japan & Food \\
\hline & $\mathrm{EU}^{* *}$ & \\
\hline \multirow[t]{4}{*}{ MON15985 × MON88913 } & Australia & Environment \\
\hline & Japan & Food/feed \\
\hline & Philippines & Food/feed \\
\hline & $\mathrm{USA}^{*}$ & Food/feed, environment \\
\hline \multicolumn{3}{|c|}{ Oilseed rape } \\
\hline \multirow[t]{7}{*}{ MS1 × Rf1 (PGS1) } & EU & Food/feed, marketing \\
\hline & Australia & Environment, food/feed \\
\hline & Canada & Environment, food/feed \\
\hline & China & Food/feed \\
\hline & Japan & Environment, food/feed \\
\hline & Korea & Food \\
\hline & USA & Environment, food/feed \\
\hline \multirow[t]{7}{*}{ MS1 × Rf2 (PGS2) } & $\mathrm{EU}$ & Food/feed \\
\hline & Australia & Environment, food/feed \\
\hline & Canada & Environment, food/feed \\
\hline & China & Food/feed \\
\hline & Japan & Environment, food/feed \\
\hline & Korea & Food \\
\hline & USA & Environment, food/feed \\
\hline
\end{tabular}


Table 4. Continued.

\begin{tabular}{lll}
\hline Crop/event & $\begin{array}{l}\text { Regulatory } \\
\text { approvals }\end{array}$ & Proposed use \\
\hline MS8 $\times$ Rf3 & EU & Food/feed, marketing \\
& Australia & Environment, food/feed \\
& Canada & Environment, food/feed \\
& China & Food/feed \\
& Japan & Environment, food/feed \\
& Korea & Food \\
& USA & Environment, food/feed \\
\hline
\end{tabular}

* USA approvals for the parental lines (source: U.S. Regulatory Agencies Unified Biotechnology Website).

** Under authorization under EC/1829/2003 (source: EU database).

view, the term "StaEv" refers to all events where multiple ModSeqs are present in the plant genome (see Fig. 4).

From the analytical point of view, the number and the structure of the integration loci are crucial. Assays to detect the transgenic event(s) are designed based on the molecular structure of the transgenic locus. Here the distinction to be made is between (1) two or more transgenes being introduced in a single locus, and (2) multiple transgenes integrated in multiple loci in the plant genome.

Introduction of two or more genes will result in different integration patterns depending on the approach used (Fig. 4). The crossing of two single transgenic events, as well as the retransformation of a single transgenic plant by Agrobacterium containing another transgene (iterative transformation strategies), will result in the combination of two different transgenes inserted at different loci in the plant genome (Ow, 2007). Iterative or multiple transformation strategies give rise to introduced transgenes that are not linked, but situated at different, randomlylocated loci in the plant's genome. As a consequence, the introduced transgenes can segregate in subsequent generations, according to Mendelian rules. Cotransformation methods are more likely to result in transgenes that co-integrate at the same chromosomal position, and that therefore may be inherited together in the progeny (Halpin, 2005).

In cotransformed events, multiple dispersed insertions have been reported along with the major insertion locus containing the different transgenes, e.g. in the case of Bt176 corn. This phenomenon is probably more common in the non-commercialized GMOs.

Although genotypically speaking, both MulEvs and StaEvs contain different transgenes, they would be treated differently when applying the OECD guidance on designation of a unique identifier. OECD (2006) prescribes design of a unique identifier for each transgenic plant, as a method of consistently identifying TraEvs in world-wide commerce. This unique identifier is a simple 9-digit alphanumeric code based on the TraEv. For plant products having two or more traits obtained through the use of recombinant DNA techniques and stacked by conventional crosses, the unique identifier is recommended to consist of the unique identifiers of each parental transgenic plant (e.g. MON-159895-7 $\times$ MON-Ø1445-2) (OECD, 2006). For example, the MulEv Bt176 corn is identified by the OECD code SYN-EV1769 (176), while the StaEv T25 $\times$ Mon810 is identified by the OECD code ACS-ZMØØ3-2 $\times$ MON-ØØ81Ø-6. Effectively, this means that while progeny of StaEvs can be accurately described by reference to their parental OECD codes if the transgenes segregate, the same is not the case with MulEvs. As a consequence, the same code may apply to substantially different transgenes.

The terminology proposed by Holst-Jensen et al. (2006) does not take into consideration events produced by retransformation and containing more than one transgene in more than one insertion locus. Again, a single OECD identifier code applies, which does not reveal the presence of multiple transgenes (MulEvs). For instance, cotton event 15985 (OECD identifier MON-15985-7) is a retransformed derivative of the MON531 cotton event with identifier MON- $\varnothing 531-6$, and contains three transgenic inserts integrated in three different sites in the genome: one complete and one incomplete copy of the crylAc insert and one copy of Cry2Ab (AgBios, 2008).

Different unlinked transgene inserts can also be present in the genome of one plant as a result of the introduction of multiple insert copies by transformation of a single ModSeq (Fig. 4). From the analytical point of view, no difference can be made between multi-copy events obtained by single transformation of a single ModSeq and GSs obtained by crossing, because the T-DNA is integrated in different loci which are physically unlinked (Holst-Jensen et al., 2006). The difference is in the phenotype: multi-copy events contain only one or a few but linked effective genes resulting in a single trait, while stacked events contain multiple traits. For this reason also we speak of single ModSeq stacks (SMSs) for the first group, and multiple ModSeq stacks (MMSs) for the second, where both groups are examples of GS (Fig. 4).

This difference between stacking of traits (phenotypic stacking) and stacking of events/genes (genotypic 


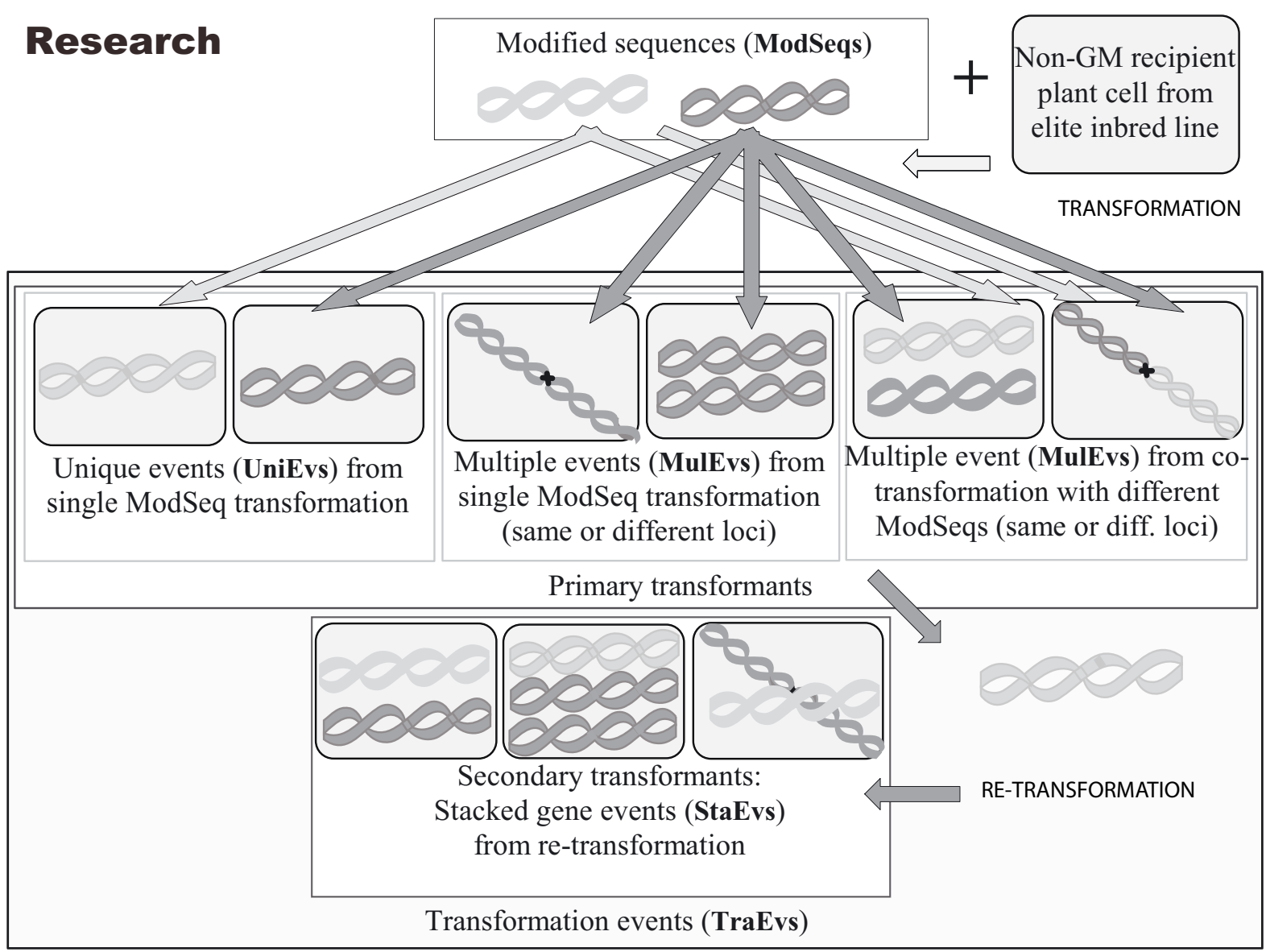<smiles>C1CCCCC1</smiles>

\section{Development}

Figure 1. Diversity of genetic origin and structure of transformation events (TraEvs) - Research stage. A ModSeq can be inserted by transformation and integrate in a single copy or in multiple copies (complete or not, rearranged or not) in one or more loci in the genome. Integration of multiple copies of a ModSeq will result in a MulEv. Depending on the definition of a ModSeq (sequence contig or single effect-gene construct) a ModSeq may include more than one effect gene, and consequently insertion of a single ModSeq may result in a multi-trait phenotype. By cotransformation, two different ModSeqs are simultaneously inserted and tend to integrate in a single locus in the genome, although multi-loci insertion is also possible. Retransformation of a single ModSeq line results in the integration of another ModSeq in a different locus. This process will create multi-trait phenotypes, which may be defined as gene-stacked (GS) events (StaEvs).

stacking) is important in the frame of analytical detection and identification of GMOs. All stacked events are GSs, however the opposite is not true. Thus, the definition of GSs used in this paper applies to all situations where multiple, but not necessarily different, genes are inserted in the plant genome.

Taking into account this duality of the term "stacking" we can also see the discrepancy between legal and analytical requirements. Seen from the breeder's point of view, in cultivation and frequently also in research and for regulatory approval purposes, stacking is interpreted in terms of combination of traits. Seen from the viewpoint of analytical detection, stacking is considered as the combination of events, an event being defined by its integration pattern in the plant genome. In the broadest sense of the word, stacking can also refer to the presence of multiple genes, including multiple genes introduced by a single ModSeq transformation. As such, a multi-copy event or SMS (Fig. 4), is not considered to be a stacked event while it is a GS. 


\section{Development}
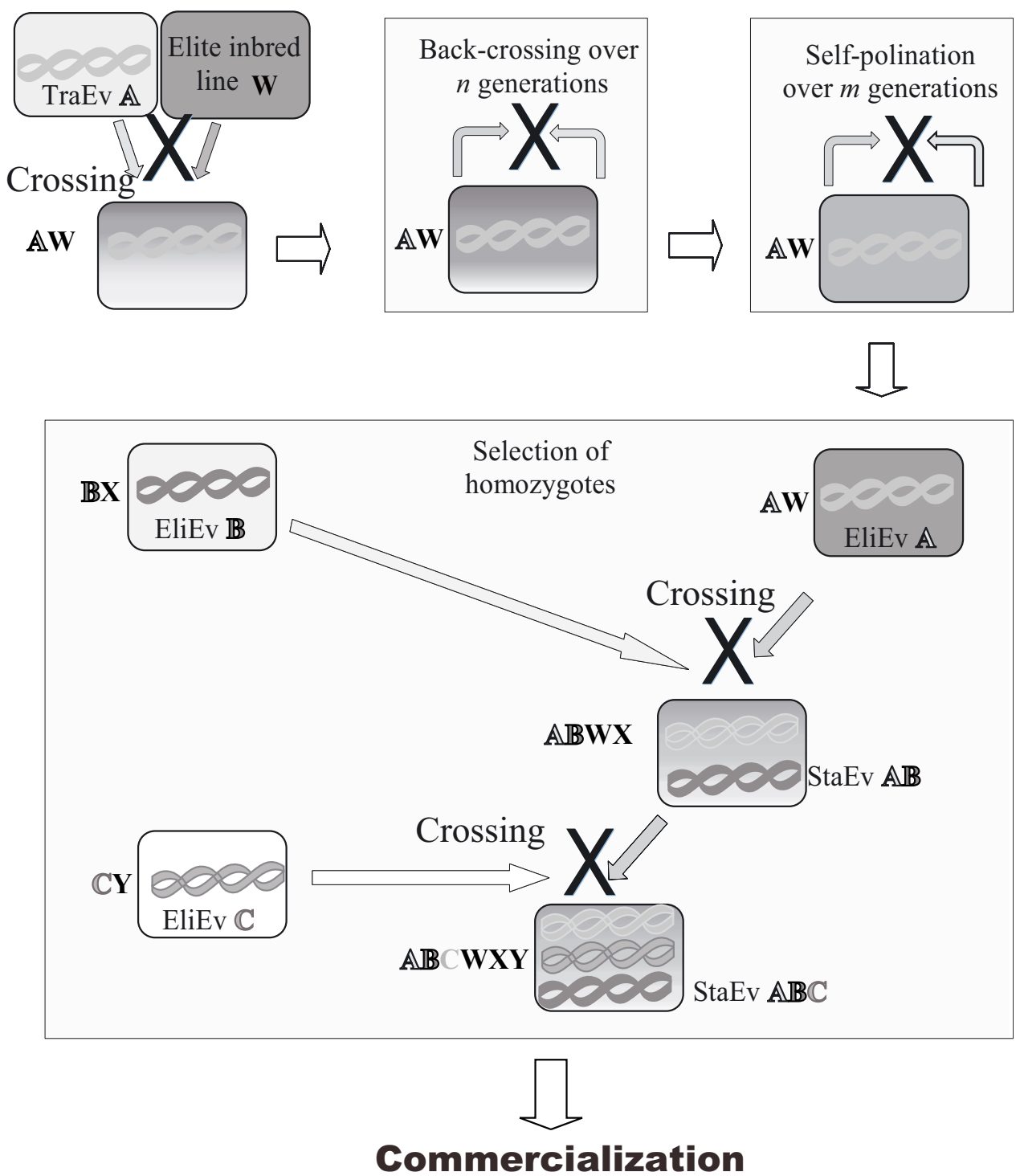

Figure 2. Diversity of genetic origin and structure of transformation events (TraEvs) - Development stage. TraEvs (Fig. 1) are usually crossed with an elite inbred (non-GM) line and then further subjected to several cycles of backcrossing and self-pollination to produce homozygous TraEvs from which those with optimum trait performance are selected (EliEv). The EliEv is subject to commercialization and regulation, and can be further crossed to produce gene stacked events (StaEvs). From the detection point of view, a MulEv cannot in principle be distinguished from a StaEv.

Besides multiple single inserts of the same construct (SMSs, Fig. 4), other types of GSs not considered so far are tandem repeat inserts and cotransformed partial constructs and rearrangements. Although multiple inserts are present in such cases, as long as they concern (parts of) the same effective set of genes, i.e. the same transgenic trait, these cases are not to be considered as stacked events. But they are also covered in the definition of GSs (Fig. 4).

\section{CULTIVATION AND REGULATION OF GENE STACKS}

\section{Global commercialization of gene stacks}

In 2007 , the total area of cultivated biotech crops had increased $22 \%$ compared to 2006 . The cultivated area of cGSs bearing two or three traits also increased. In the USA, $37 \%$ of the biotech crops conferred double 


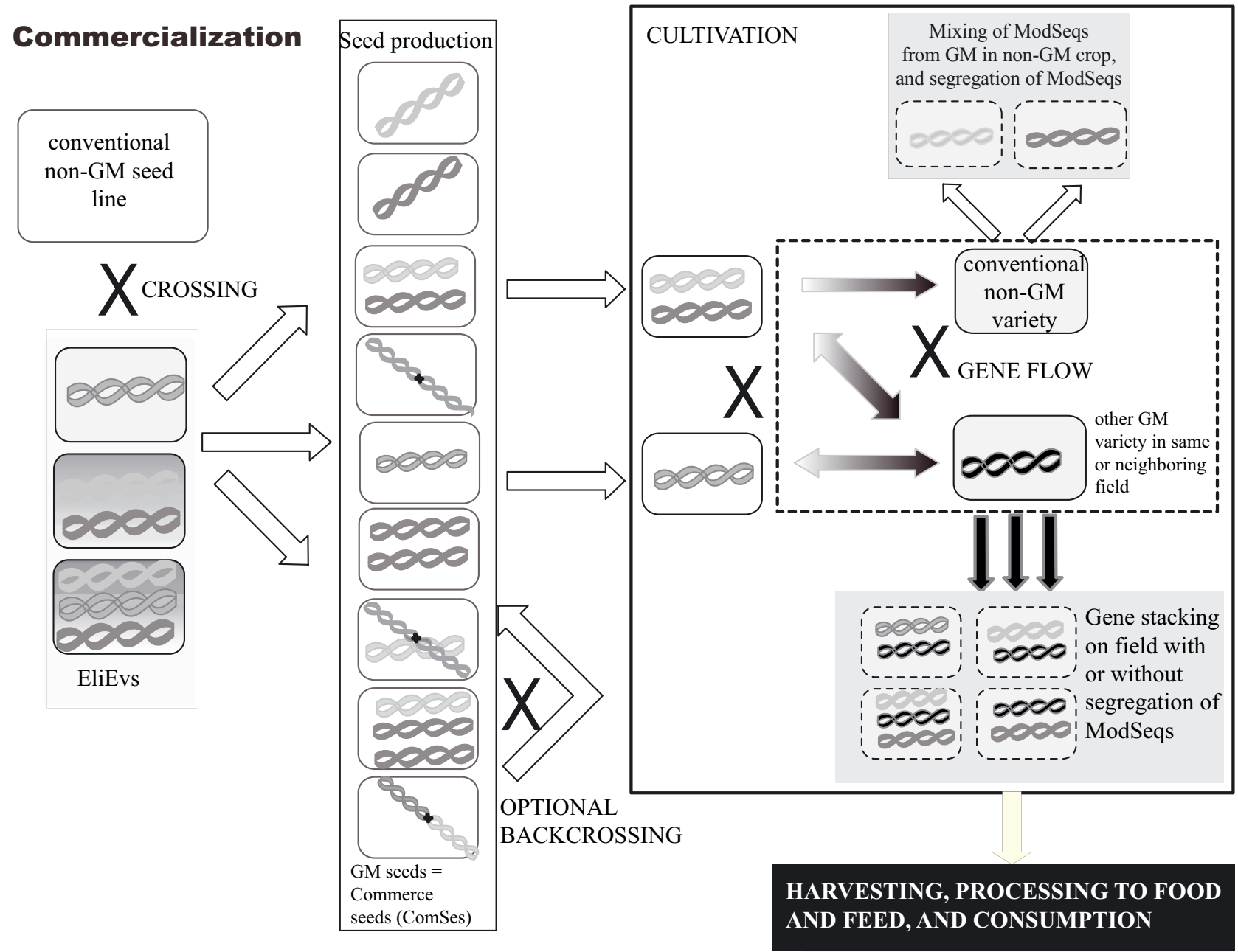

Figure 3. Diversity of genetic origin and structure of transformation events (TraEvs) - Commercialization (post-marketing) stage. The EliEvs (Fig. 2, including StaEvs) are crossed with a conventional (non-GM) seed line to produce commercial seeds (left), further used for cultivation and production of products for food and feed purposes. Depending on the crop, the commercial seeds can contain the ModSeq in homo- or heterozygous state. As a result of cultivation (right), unintended gene stacking can occur, due to gene flow from one transgenic plant to another. This cycle of unintended gene stacking can be repeated, and plants containing more than two stacked traits can occur. 


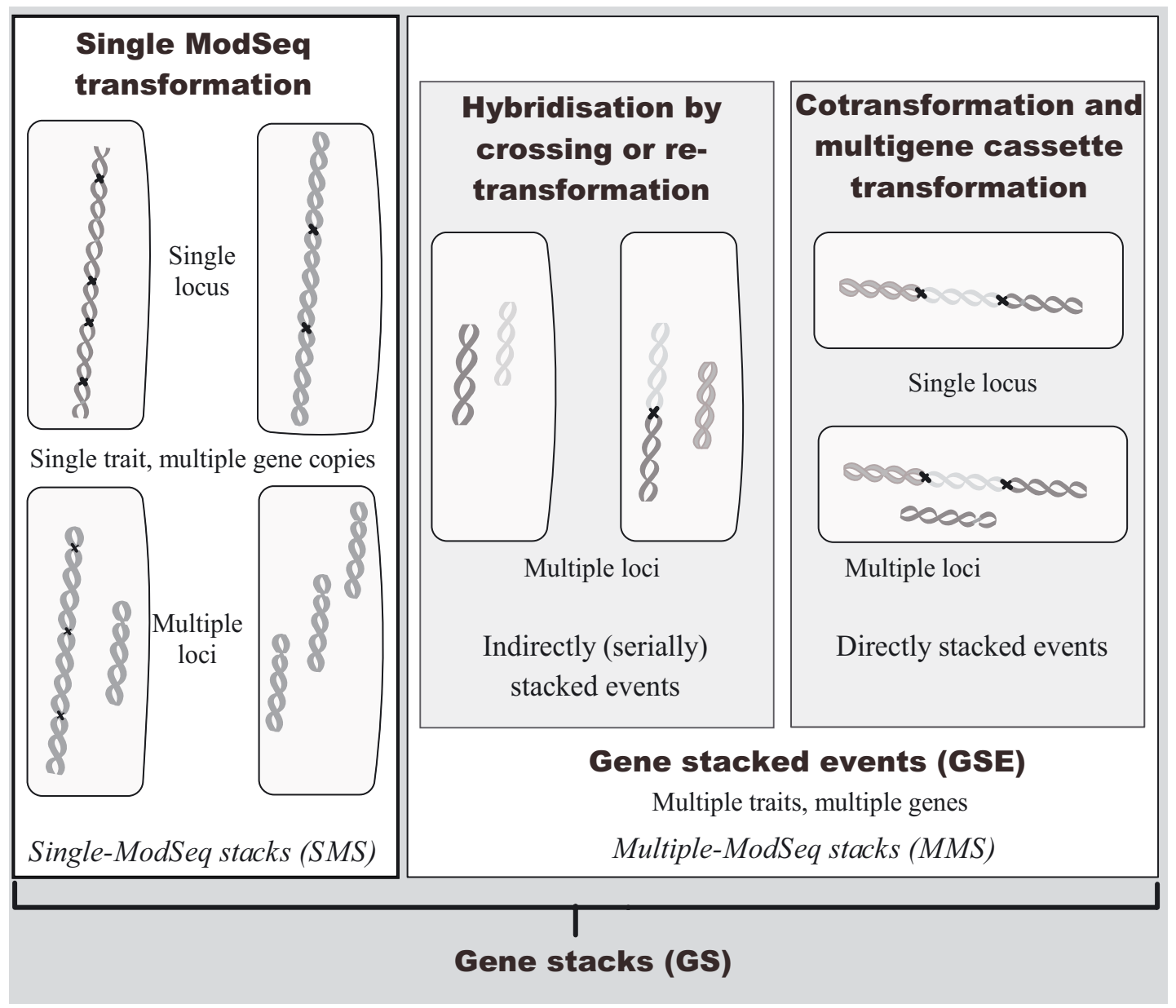

Figure 4. Terminology for gene stacking, seen from the analytical detection viewpoint. Molecular structure of the integration locus/loci, resulting from different ways to produce GSs. Multiple T-DNA copies can be introduced as a result of the transformation of a single transgene. The "single ModSeq stacks" (left part) confer a single trait inserted in multiple copies in a single or multiple loci. A single transformation process (e.g. cotransformation) can lead to multiple dispersed and/or partial insertions of (parts of) the same gene. Lines produced by cross hybridization and retransformation will contain different transgenes that are physically unlinked (middle part). Cotransformation and transformation with multiple gene cassettes often lead to integration of different T-DNAs into a single locus in the host genome (right part). Analytically, neither of the latter two categories of GS can be detected with only one transformation-event-specific PCR assay. Both single-ModSeq stacks (SMSs, left) and multiple-ModSeq stacks (MMSs, middle and right) contain multiple genes or parts of genes, and therefore can be categorized as gene stacks (GSs). See also Figures 1-3 for explanation of symbols.

herbicide tolerance or/and multiple insect resistances, while $68 \%$ of the transgenic corn and $78 \%$ of the transgenic cotton in USA have stacked traits (James, 2007).

Expected trends for the next decade of biotech crop cultivation are: an increasing area planted with biotech crops with stacked traits, development of a new generation of transgenic crops with stacked traits, and the introduction of multiple genes involved in multiple metabolic pathways (metabolic engineering). While today, cross-hybridization, retransformation, transformation with multigenic cassettes and cotransformation are the main ways of stacking several traits, new multipletrait stacking technologies are now being announced. An example is the SmartStax ${ }^{\mathrm{TM}}$ technology from Monsanto and Dow (Monsanto, 2007), which aims at an eightgene stack in corn, combining insect resistance (IR) traits Herculex (two, Dow) and YieldGard (two, Monsanto) and herbicide tolerance (HT)-weed control systems Roundup Ready and LibertyLink ${ }^{\mathrm{TM}}$ (Monsanto). Also, new multigenic cassette assembly methods and plant transformation vectors e.g. polycistronic transgenes, are being deployed (Halpin, 2005; Shrawat and Lörz, 2007). 


\section{Intended versus unintended gene stacking}

In the context of the EU regulations, it is useful to make a distinction between intended and unintended GSs (uGSs, Fig. 3). The former are intentionally produced and subject to authorization and commercial use, whilst the latter will accidentally appear on or around the cropping areas with a frequency defined by the potential for transgene stacking by crossing. Consequently, the impact of cGS and uGS will depend on the relative abundancies of GM events planted in the relevant environment. Furthermore, uGSs are formed only in circumstances that allow close contact between transgenic plants carrying different transgene traits. Establishment and persistence of uGSs will depend on certain crop-specific factors, such as the biology of the plant, reproductive system, pollen and seed dispersal, occurrence of volunteers, ecology of the species and the agricultural practices.

The creation of an uGS may result from gene flow from transgenic plant to transgenic plant followed by formation of seeds that remain in the soil and grow in the next cultivation period. Volunteers appear to be a major source of uGSs in oilseed rape (Messéan et al., 2007). The spontaneous on-field crossing of two oilseed rape transgenic events can evolve to multiple resistances and occur in the harvest at frequencies much higher than the EU threshold for labeling (Messéan et al., 2007), provided that selection is driving the process. Controlling stacked HT oilseed rape volunteers might not be significant in certain circumstances, e.g. in the UK the use of glufosinate is presently very limited, and stacking of glufosinate and glyphosate tolerance genes will have no additional practical significance. The stacked herbicide volunteers could be a problem when they need to be controlled in other HT crops, such as corn and sugar beet, which could change the cultural and chemical practices (Orson, 2002).

GSs can occur as a result of intraspecific crossing of transgenic plants persisting in feral populations out of the cropped land. In Japan, where no oilseed rape is cultivated, GSs has been observed in feral populations (Aono et al., 2006; Saji et al., 2005). The origin of these uGSs is not clear, but it could occur either as result of spontaneous cross-hybridization or via seed dispersal of imported stacked seeds (Aono et al., 2006).

\section{Legal and regulatory aspects of gene stacks}

Gene stacking is regulated differently in different countries. A cGS produced as a result of traditional plant breeding with transgenic parents is not automatically subject to regulation in all jurisdictions, contrary to the individual parental lines. In the USA and Canada, stacks are considered as products of conventional breeding, with the presumption of a positive harmless biosafety assessment.
Registration is only needed upon identification of a specific hazard, e.g. synergistic effects of combining various insecticidal proteins. In the EU to the contrary, each cross between two or more transgenic lines is considered as a new GMO event, which needs to be assessed and approved, even though individual parental events may have market approval. Here risk assessment is focused on possible additional effects. The EFSA Guidelines apply to stacks obtained by cross hybridization (see also Definitions related to risk assessment, above).

Between these two extreme types of jurisdictions for stacks, intermediate approaches exist. In the latter, registration is needed, however the product benefits from the presumption of safety. Limited data showing that the product behaves as expected is then the major data requirement (unless a specific hazard is identified). Furthermore, there is a distinction between deliberate release into the environment, and use in food/feed. In relation to labeling, this distinction is particularly relevant, because detection methods used to ensure compliance with labeling regulations have very significant limitations in relation to GSs.

The main issue in all GM legislation is whether or not the GMO product is safe, so the first question in relation to GSs is therefore if the jurisdiction considers that a GS has the potential to result in an unsafe product, when the parent GMOs are considered safe. If this is the case, then separate risk assessment of the cGS is required. In some jurisdictions, this is considered on a case-by-case basis, e.g. Australia, Brazil, Canada, China, New Zealand and USA (AgBios, 2008; CFIA, 2004; CMOA, 1996; CTNBio, 2005; FDA, 1992; OGTR, 2000, 2007), and whether the cGS formally becomes authorized or not may vary. Advance notification prior to release is always required in some jurisdictions, e.g. Argentina, Canada, EU, Japan, Korea and the Philippines (AgBios, 2008; CFIA, 2004; EC, 2001; SAGPyA, 2003). Despite the recommendations in a recent discussion paper, specific requirements for provision of detailed information permitting a safety assessment of the cGS are not included in the amendments of the Australian Gene Technology Regulations (OGTR, 2007).

\section{DETECTION OF GENE STACKS}

\section{Specific issues related to GS identification and quantification}

If the sole purpose of testing is to determine if GM material is present, then it may not be necessary to test for stacking. However, if for instance the GS is legally considered distinct from the parental GMOs, and it is necessary to discriminate between authorized and unauthorized GM material, then identification of stacked material 


\section{Taverniers et al.}

may immediately become necessary. Specific detection and quantification methods are available for most commercialized UniEv GMOs, and these methods may be used to identify and quantify cGSs. However, these methods are limited by their inability to discriminate between mixtures of GMOs, such as a 50:50 (\%) mix of two separate GM events (the parents of the stack), and a 50:50 (\%) mix of non-GM and the cGS event, or mixtures of the single transgenic lines and stacked event (as it will segregate in F2 seeds of the GS). Such discrimination will only be achievable if the single TraEvs can be shown to have a non-independent distribution (co-occurrence). This is easily achieved on a single seed, but not in a processed product like flour.

First, labeling requires identification, and this comes down to unambiguous, unique, transformation-eventspecific identification. With the current EU legislation, the authorization of a StaEv obtained by crossing single events goes through separate authorization procedures (e.g. double stack: two validated event-specific methods needed), regardless of the authorization status of the individual lines.

It is impossible to define a unique molecular marker for a StaEv that will be vertically transmitted along with the ModSeqs. As a consequence, the detection method for a StaEv is currently a combined set of event-specific detection methods for each individual ModSeq. Related to this is the choice of certified reference materials (CRMs) to use for GS detection: If the above is sufficient, then individual, single-event CRMs may be used for the purpose of identification of GSs.

Second, labeling on the basis of a threshold implies a need for quantitative methods. Many countries require labeling of GM products if the GM share of a single ingredient exceeds a specific threshold: e.g. $0.9 \%$ in the EU, $1 \%$ in Australia and New Zealand, 3\% in Korea, and 5\% in Japan and Indonesia (Carter and Gruere, 2003). Labeling of GM material is required, provided that the quantity exceeds such a defined threshold, and that the material is derived from authorized GMOs. For unauthorized GMOs, the tolerance is much lower, but not necessarily well defined. When the GM content of a harvest or any GM product is being measured, GSs may have a strong influence on the measured GM content (see Holst-Jensen et al., 2006 for more details). Compared to a weightor particle- (seed/kernel)-based approach, one based on the holoploid genome (DNA content of the whole chromosome complement) may result in overestimates of the GMO quantity, since the presence of two ModSeqs in a single holoploid genome would yield an estimated GMO concentration of $200 \%$. On the other hand, a hemizygous single UniEv derived GM corn seed will yield an estimated GMO concentration of only $50 \%$. In contrast, if seed is the prevailing unit, the same seed is deemed $100 \%$
GM. Thus, there will be cases where the measured GMO content is lower and cases where it is higher depending on the approach applied. The main difference, according to Holst-Jensen et al. (2006) is whether the applied approach is consistent and coherent or not.

- It is presently not clear if authorized cGSs need to be quantified separately from the parental GMOs in those jurisdictions where GM material labeling is required. However, farmers need to know the characteristics of the seeds that they sow, so consequently seed traders need to be able to identify and quantify GSs in the seeds.

- As pointed out by Holst-Jensen et al. (2006), a full implementation based on measurements of haploid genome equivalent (HGE) will have clear consequences for the resulting GM quantity determination, since GSs contain more ModSeqs than UniEvs. Analytical methods can only measure analytes, and these can, within certain limits, be traced throughout the food/feed chain. Seed- or mass-based units are not traceable after processing that involves grinding of the seed. Consequently, from a scientific point of view, the only traceable and coherent unit is the HGE unit, despite political and historically based opposition.

- Another important issue is to consider in how far the distinction in types of gene/event stacks, as described above, needs to be made in relation to quantification of the stacks. Should a quantitative analysis of a MulEv obtained by cotransformation be treated differently from a quantitative analysis of a StaEv, if the ModSeqs in both events are the same? As explained above, in both cases the ModSeqs are physically unlinked, thus per definition and from an analytical viewpoint, there is no difference (Holst-Jensen et al., 2006). From a detection point of view, it was argued that the most coherent approach would be to treat StaEvs and MulEvs equally, and that the GM quantity for all GM materials should be measured and reported with reference to quantity of ModSeqs per HGE.

- We could extend this issue to the question: What is the impact on quantification of the technique of transformation (e.g. only nuclear DNA is transformed versus transformation of extranuclear DNA) and the way of production of the GS (e.g. MulEv versus StaEv but also, MulEv versus single-transformed multi-copy event, see Figs. 1-3 and Fig. 4). Analytically it may also be impossible to distinguish between uGS and cGS. Table 5 serves to illustrate the analytical challenge. Analytically, based on DNA, there is a significant difference between measuring $\geqslant 3$ ModSeqs (Bt176), 2 ModSeqs (stacked T25 $\times$ MON810) and 1 ModSeq (T25 or MON810) per mass or particle 
unit. It may be desirable to clarify in regulations how GSs shall be treated in a more coherent manner. We recommend following Holst-Jensen et al. (2006). This is also in line with the core of EC Recommendation 787/2004 (EC, 2004b).

\section{Existing methods for GS identification and quantification}

As mentioned above, transformation-event-specific detection methods exist for most UniEvs. Official, internationally validated PCR-based methods are available, e.g. from the website of the EU Community Reference Laboratory for GMOs (http://gmo-crl.jrc.it/). Application of the individual ModSeq-event-specific methods for identifying the individual ModSeqs or genes in GSs, has the limitation that distinction cannot be made between a mix of two separate GMOs (parents of a stack) and a mix of the cGS with non-GM. The only currently available way to distinguish mixtures of parental TraEvs and StaEvs (containing the same ModSeqs) is to analyze the single individual plant or fruit. Several methods relying on single-kernel-based analysis either by using multiplex real-time PCR (Akiyama et al., 2005) or protein flow strips (Ma et al., 2005) are described. However, singlekernel-based analysis is appropriate only if limited numbers of seeds have to be analyzed. Allnut et al. (2006) developed a protocol for detection of GSs in seed pools by combining a sub-sampling strategy (control plan by multiple attributes, Laffont et al., 2005) with real-time PCR for detection of Ms8xRf3. The basic idea is to detect both event-specific sequences plus the bar gene which occurs in both events. The segregation pattern of the markers would then give an indication of the presence and abundance of the GS.

Other approaches based on analysis of seed pools are under development. Kobilinsky and Bertheau (2005) developed a subsampling approach (control plans by multiple attributes) for GMO detection by application of qualitative testing methods, taking into account the cost of analysis. This method is not directly applicable to GSs, but V. Ancel (INRA, Co-Extra, personal communication) is exploring an approach for commercial GS detection based on qualitative PCR.

\section{Other remarks}

Currently, all commercial GM plants carry their ModSeq in the nuclear DNA. As a consequence, the inserted sequences of all commercial GMOs are transferred to successive generations in a Mendelian manner, a biosafety and breeding requirement. What has not been considered so far is the situation where the ModSeq is inserted in the
DNA of organelles (chloroplasts, mitochondria). In this case, the transgenic DNA would be inherited mostly maternally, and quantitative relationships may be more obscure, since the organelles are often multicopy, with variable number per cell in different tissues of an organism, and because each organelle contains a variable number of copies of its genome. Plastid transformation is considered as a possible means of reducing unintended dissemination of transgenes via pollen (Ruf et al., 2007).

\section{CONCLUSIONS}

The following are our main conclusions and identified remaining gaps, future needs and recommendations regarding:

- Terminology and definitions: Related to the different techniques of transformation and production of stacks, including classical breeding, and to differences in global authorization and commercialization practices, we conclude that a uniform definition of gene stacking is lacking. Stacking can primarily be explained in terms of the stacking of traits, the stacking of events, and in the broadest sense, the stacking of genes. Gene stacking, as the subject of this paper, covers all types of stacking, including the combination or presence of multiple copies of a single modified sequence introduced in the plant genome as a result of the initial transformation. Distinction is made between different types of stacks, according to the way they are produced. A general terminology is proposed in Figures 1-3 and more particularly in Figure 4 . We thus specifically point out the distinction between stacking of phenotypes (traits) and stacking of gene sequences (copies).

- Unintended gene stacks: Taking suitable measures to limit the gene flow between transgenic fields, the formation of unintended GSs can be limited, and the persistence of stacked-gene volunteers can be minimized or practically avoided by application of suitable crop-specific agricultural practices. This may be of less relevance where no GS is cultivated and where the cultivation of transgenic plants is limited. Unintended GSs can occur in feral populations as a result of seed dispersal during transportation. Measures to manage feral populations could be of more interest in the future to avoid persistence of GS volunteers.

- Detection and quantification of GSs: Analytical methods to detect GSs are primarily seed-based detection strategies, making use of event-specific PCR assays or protein assays. For quantification, real-time PCR is the method of choice. Detection of GSs in seeds is possible, but approaches are needed that can be used on a routine basis. Suitable approaches relying 
Table 5. Comparison of "unique events" (UniEv), "stacked events" (StaEv) and "multiple events" (MulEv) in relation to their DNA content.

\begin{tabular}{lllll}
\hline $\begin{array}{l}\text { Type of } \\
\text { GMO }\end{array}$ & $\begin{array}{l}\text { OECD unique } \\
\text { identifier }\end{array}$ & $\begin{array}{l}\text { Popular name } \\
\text { of GMO }\end{array}$ & ModSeq & $\begin{array}{l}\text { Copy number in } \\
\text { the GMO }\end{array}$ \\
\hline UniEv & ACS-ZMØØ3-2 & T25 corn & PAT & 1 \\
\hline UniEv & MON-ØØ81Ø-6 & MON810 corn & CryIAb & 1 \\
\hline StaEv & ACS-ZMØØ3-2 $\times$ & Stacked T25 $\times$ & PAT & 1 \\
& MON-ØØ81Ø-6 & MON810 corn & CryIAb & 1 \\
\hline MulEv & SYN-EV176-9 & Bt176 corn & PAT & $\geqslant 1^{\mathrm{b}}$ \\
& $(176)$ & & CryIAb & $\geqslant 2^{\mathrm{b}}$ \\
\hline
\end{tabular}

\footnotetext{
a The copy number in the GMO corresponds to the number of analytes contributed to a DNA-based analytical test per haploid genome of the GMO. Protein content may vary, due to differences in expression levels between lines, individual plants and to abiotic conditions. In bioassays, the trait is only detectable if the expression level is high enough to induce an observable biological effect, and if the biocide has the desired effect on the target organism.

${ }^{b}$ Present information on the AgBios database (AgBios, 2007) does not specify the number of copies present in the GMO. Earlier reports from the developer stated that each gene is present in 2-5 copies in the transformed plant genome. It is also possible that the number of copies varies between commercial seed lines on the market, since variations of sequences have been observed, for instance between Pactol and Garona cultivars (Y. Bertheau, unpublished).
}

on analysis of sub-samples/pools of seeds and realtime PCR-based detection methods allowing to distinguish between stacks and their parental lines would be the ideal solution for routine application in GMO testing laboratory.

- Global regulation of GSs: Although the key principle of all GM legislations worldwide is the issue of substantial equivalence compared to its conventional counterpart, and thus the safety of the GMO, GSs are regulated differently in different countries. In Canada and the US, stacks are considered as conventional breeding products with a presumption of biosafety assessment. In other jurisdictions, GSs must be registered, but not assessed for safety. In the EU, finally, even if two parental GM lines have market approvals, the cross between them is considered as a new event, subject further to the same biosafety assessment and approval rules.

- Compliance between EU regulations and detection of GSs: Regulations in place within the EU are comprehensive with respect to defining the basis for identification and quantification of GM materials. The definition of transformation "event" is essentially the definition of what to identify/quantify. However, of high relevance and underlying the political decisions is the question whether the regulations shall be scientifically coherent or not. Coherence in regulations would require first that the EU decide what the prevailing unit of measurement and expression throughout the food/feed chain shall be. Various stakeholders could refer to seeds/kernels, masses, volumes, haploid (holoploid or monoploid) genome equivalents
(HGE, DNA based) or protein equivalents. Second, coherence would require that the unit be applied consistently, without ad hoc exceptions. A consistent application of the HGE-based unit of measurement and expression will lead to GS materials being generally assigned a higher GM content than non-GS materials. The advantages of this approach will, in our opinion, clearly outweigh the drawbacks. In particular, this approach may permit predictions and traceability of the GM content of products all the way from seed to fork, once the GM content of the seed is known, with the uncertainty parameters being limited to sources of contamination (e.g. pollen influx, volunteers in the fields and insufficient cleaning of transport, storage and production equipment and containers). Without a clearly defined and prevailing unit of measurement and its use through the entire food/feed chain, including seeds, there will always be room for legal dispute between stakeholders applying different measurement/expression units. In our opinion, regulatory systems should primarily be practical, consistent and preferably science-based. We believe that the only scientifically justifiable solution is to formally adopt HGE as the legally correct unit of measurement/expression of GMO content, and that this is the only consistent alternative throughout the food/feed chain. Although it may introduce some practical difficulties, in particular for high-throughput analytical testing, this alternative is largely also practical, since it may reduce the need for analytical testing and pave the road for more document-based traceability from seed to fork. 


\section{ACKNOWLEDGEMENTS}

This work was financially supported by the European Commission through the Sixth Framework Program, integrated project Co-Extra (http://www.coextra.eu), contract FOOD-2005-CT-007158) and by the Research Council of Norway (grant no. 170363/D10).

Received June 29, 2007; accepted July 24, 2008.

\section{REFERENCES}

AgBios (2008) AgBios, Ontario, Canada. GMO database. http://www.agbios.com/dbase.php (accessed July 1st 2008)

Akiyama H, Watanabe T, Wakabayashi K, Nakade S, Yasui S, Sakata K, Chiba R, Spiegelhalter F, Hino A, Maitani T (2005) Quantitative detection system for corn sample containing combined-trait genetically modified corn. Anal. Chem. 77: $7421-7428$

Allnutt T, McMillan J, McArthur R, Henry C (2006) VS0136; A combined protocol for PCR Detection of GM in Seed. CSL, York, UK.

http://www.gm-inspectorate.gov.uk/documents/ COPsFinalReportweb_000.pdf (accessed July 1st 2008)

Aono M, Akiyama S, Nagatsu M, Nakajima N, Tamaoki M, Kubo A, Saji H (2006) Detection of feral transgenic oilseed rape with multiple herbicide resistance in Japan. Environ. Biosafety Res. 5: 77-87

Carter CA, Gruere GP (2003) International approaches to the labeling of genetically modified foods. Choices 18: 1-4. http://www.choicesmagazine.org/2003-2/2003-2-01.htm (accessed July 1st 2008)

CFIA (2004) Canadian Food Inspection Agency, Plant Products Directorate, Plant Biosafety Office. Directive 94-08 (Dir9408), Assessment Criteria for Determining, Environmental, Safety of Plants With Novel Traits. http://www.inspection. gc.ca/english/plaveg/bio/dir/dir9408e.pdf (accessed July 1st 2008)

CMOA (1996) Ministry of Agriculture, People's Republic of China. Order No. 7, the safety administration implementation regulation on agricultural biological genetic engineering. http://www.cbi.pku.edu.cn/mirror/binas/Regulations/full_ regs/china/c2.htm (accessed March 1st 2007)

CTNBio (2005) National Technical Commission on Biosafety, Brazil. Implementation of the provisions of the biosecurity law 11.105. http://www.ctnbio.gov.br/index.php/content/ view/3670.html?toPrint=yes (accessed July 1st 2008)

Cummins J (2004) GM rice in India. ISIS press release, 30/11/2004. http://www.i-sis.org.uk/GMRII.php (accessed July 1st 2008)

De Schrijver A, Devos Y, Van Den Bulke M, Cadot P, De Loose M, Reheul D, Sneyers M (2007) Risk assessment of GM stacked events obtained from crosses between GM events. Trends Food Sci. Technol. 18: 101-109
EC (2001) Directive 2001/18/EC of the European Parliament and the Council on the deliberate release into the environment of genetically modified organisms and repealing Council Directive 90/220/EEC. Off. J. Eur. Union 106: 1-38

EC (2003a) Regulation 1829/2003/EC on genetically modified food and feed. Off. J. Eur. Union 268: 1-23

EC (2003b) Regulation (EC) 1830/2003 concerning the traceability and labeling of genetically modified organisms and the traceability of food and feed products produced from genetically modified organisms and amending Directive 2001/18/EC. Off. J. Eur. Union 268: 24-28

EC (2004a) Commission regulation (EC) No 641/2004 on detailed rules for implementation of Regulation (EC) No 1829/2003 of the European Parliament and the Council as regards the application and authorization of new genetically modified food and feed, the notification of existing products and adventitious and technically unavoidable presence of genetically modified material which has benefited from a favorable risk evaluation. Off. J. Eur. Union 102: 14-25

EC (2004b) Commission recommendation 2004/787/EC on technical guidance for sampling and detection of genetically modified organisms and material produced from genetically modified organisms as or in products in the context of Regulation (EC) No 1830/2003. Off. J. Eur. Union 348: 18-26

EFSA (2004) European Food Safety Authority. Guidance document of the scientific panel on genetically modified organisms for the risk assessment of genetically modified plants and derived food and feed. The EFSA J. 99: 1-94

EFSA (2006) European Food Safety Authority. Public consultation of the document "Risk assessment of plants containing genetic modification events combined crossing". http:// www.efsa.europa.eu/en/science/gmo/gmo_consultations/ gmo_hybrids_publcons.html (accessed April 10th 2007)

FDA (1992) Food and Drug Administration, USA. Statement of policy: foods derived from new plant varieties; notice. Federal Register 57 (104): 22983-23001. http://www.agbios.com/ docroot/articles/01-319-004.pdf (accessed March 1st 2007)

Halpin C (2005) Gene stacking in transgenic plants - the challenge for 21 st century plant biotechnology. Plant Biotech. J. 3: $141-155$

Holst-Jensen A, De Loose M, Van den Eede G (2006) Coherence between legal requirements and approaches for detection of genetically modified organisms and their derived products. J. Agric. Food Chem. 54: 2799-2809

James C (2007) Global status of commercialized biotech/GM crops in 2007. ISAAA Brief 37: Executive Summary

Kobilinsky A, Bertheau Y (2005) Minimum cost and acceptance sampling plans for grain control, with application to GMO detection. Chemom. Intell. Lab. Syst. 75: 189-200

Laffont J-L, Remund KR, Wright D, Simpson RD, Gregoire S (2005) Testing for adventitious presence of transgenic material in conventional seed or grain lots using quantitative laboratory methods: statistical procedures and their implementation. Seed Sci. Res. 15: 197-204 
Lezaun J (2006) Creating a new object of government: making genetically modified organisms traceable. Soc. Stud. Sci. 36: 499-531

Ma BL, Subedi K, Evenson L, Stewart G (2005) Evaluation of detection methods for genetically modified traits in genotypes resistant to European corn borer and herbicides. $J$. Environ. Sci. Health. Part B 40: 633-644

Messéan A, Sausse C, Gasquez J, Darmency H (2007) Occurence of genetically modified oilseed rape seeds in the harvest of subsequent conventional oilseed rape over time. Europ. J. Agronomy 21: 115-122

Monsanto (2007) Monsanto - Dow Agrosciences Collaborative Agreement SmartStax: New industry-standard stacked-trait platform, Sept. 14, 2007. http://www.monsanto.com/pdf/ investors/2007/09-14-07.pdf

OECD (2006) Organisation for Economic Co-operation and Development. OECD Environment, Health and Safety Publications, Series on Harmonisation of Regulatory Oversight in Biotechnology, no. 23. Revised 2006: OECD guidance for the designation of a unique identifier for transgenic plants, ENV/JM/MONO(2002)7/REV1.

http://www.olis.oecd.org/olis/2002doc. nsf/ENGREFCORPLOOK/NT000059E2/

$\backslash$ protect $\backslash \mathrm{T} 1 \backslash$ textdollarFILE/JT03217233.PDF $\quad$ (accessed July 1st 2008)

OGTR (2000) Office of the Gene Technology Regulator of Australia. The Gene Technology Act 2000. AttorneyGeneral's Department, Canberra, Australia. http://scaletext. law.gov.au/html/pasteact/3/3428/top.htm (accessed July 1st 2008)
OGTR (2007) Office of the Gene Technology Regulator of Australia. Compilation of the Gene Technology Amendment Regulations 2006 with the Gene Technology Regulations 2001 (commencement date 31 March 2007, subject to disallowance). http://www.ogtr.gov.au/pubform/legislation.htm (accessed July 1st 2008)

Orson J (2002) Gene stacking in herbicide tolerant oilseed rape: lessons from the North American Experience. English Nature Research Reports 43, ISSN 0967-876X. http://www. agbios.com/articles.php (accessed July 1st 2008)

Ow DW (2007) Site-specific recombination for plant genetic engineering: Strategy for agro-mediated gene stacking. Proc. of the Intern. Symp. on Biotechnol. of Temperate Fruit Crops and Trop. Spec. 738: 117-127. In Litz RE, Scorza R, eds, Acta Horticulturae, Book series, 2007

Ruf S, Karcher D, Bock R (2007) Determining the transgene containment level provided by chloroplast transformation. Proc. Natl. Acad. Sci. USA 104: 6998-7002

SAGPyA 39/03 (2003) Application for permit to release genetically modified plant organisms into the environment. http:// www.agbios.com/docroot/decdocs/06-325-001.pdf (accessed July 1st 2008)

Saji H, Nakajima N, Aono M, Tamaoki M, Kubo A, Wakiyama S, Natase Y, Nagatsu M (2005) Monitoring the escape of transgenic oilseed rape around Japanese ports and roadsides. Environ. Biosafety Res. 4: 217-222

Shrawat AK, Lörz H (2007) Agrobacterium-mediated transformation of cereals: a promising approach crossing barriers. Plant Biotechn. J. 4: 575-603 